Virtual social environments as a tool for psychological assessment: Dynamics of interaction with a virtual spouse

\author{
Felix D. Schönbrodt and Jens B. Asendorpf \\ Humboldt University Berlin, Germany
}

\title{
Psychological Assessment, in press
}

APA copyright notice: This article may not exactly replicate the final version published in the APA journal. It is not the copy of record. (http://www.apa.org/journals)

\section{Author Note}

Felix D. Schönbrodt, Department of Psychology, Humboldt University Berlin, Germany; Jens B. Asendorpf, Department of Psychology, Humboldt University Berlin, Germany.

Felix D. Schönbrodt is now at Department of Psychology, Ludwig-MaximiliansUniversity Munich, Germany.

We thank Birk Hagemeyer for his helpful and detailed comments on earlier versions of this paper.

Correspondence concerning this article should be addressed to Felix Schönbrodt, Leopoldstr. 13, 80802 München, Germany. Email: felix.schoenbrodt@web.de 


\begin{abstract}
Computer games are advocated as a promising tool bridging the gap between the controllability of a lab experiment and the mundane realism of a field experiment. At the same time, many authors stress the importance of observing real behavior instead of asking participants about possible or intended behaviors. In this article we introduce an online virtual social environment, which is inhabited by autonomous agents including the virtual spouse of the participant. Participants can freely explore the virtual world and interact with any other inhabitant, allowing the expression of spontaneous and unprompted behavior. We investigated the usefulness of this game for the assessment of interactions with a virtual spouse and their relations to intimacy and autonomy motivation as well as relationship satisfaction with the real life partner. Both the intimacy motive and the satisfaction with the real world relationship showed significant correlations with aggregated in-game behavior, which shows that some sort of transference between the real world and the virtual world took place. In addition, a process analysis of interaction quality revealed that relationship satisfaction and intimacy motive had different effects on the initial status and the time course of the interaction quality. Implications for psychological assessment using virtual social environments are discussed.
\end{abstract}

Keywords: virtual environment, motivational dynamics, intimacy motive, romantic relationships, transference 
Virtual social environments as a tool for psychological assessment: Dynamics of interaction with a virtual spouse

In a perfect psychological study, the researcher is able to maximize both external and internal validity. In reality, however, psychological studies often weight one of these two validity criteria more than the other. On the one extreme, an experiment can be set up in a laboratory with highly controllable conditions and clear inferences of causal relations, but with a highly artificial environment that reduces external validity. On the other extreme, one can perform a field study with high ecological validity but low controllability and internal validity. Computer games and virtual environments occupy an intermediate position and are advocated as promising tools in psychological research and assessment to bridge both extremes, providing a unique combination of both mundane realism and experimental control (Blascovich, Loomis, Beall, Swinth, Hoyt, \& Bailenson, 2002). Therefore, the investigation of human behavior in a naturalistic, virtual social environment (VSE) is promising where all characters behave and interact under experimental control. Other characters are implemented as active, autonomous agents, with which participants can interact. Although some researchers already investigated social behavior in virtual environments (e.g., Frey, Blunk, \& Banse, 2006; McCall, Blascovich, Young, \& Persky, 2009), to our knowledge no such study has been conducted with autonomous agents that allow rather rich social interactions. From an implementation of a VSE we expect two main advantages: (a) an effortless observation of behavior with automatic logging, and (b) a rather implicit measurement that operates on a contextually embedded and naturalistic level (in contrast, e.g., to reaction times in priming studies or Implicit Association Tasks, Greenwald, McGhee, \& Schwartz, 1998). Using VSEs, research scenarios can be tackled that can hardly be solved with conventional methods like self-report measures, laboratory studies, or interviews.

This article has two objectives: on the one hand, we want to introduce VSEs as a 
general tool for the examination of social interactions, and we report considerations which guided us in the implementation of "Simoland", a VSE which was built to investigate a participant's behavior towards his or her virtual spouse. On the other hand, we illustrate the potentials and limits of such an approach by an empirical study investigating how interpersonal motives and relationship satisfaction shape the behavior in Simoland..

In the remainder of the article we (a) discuss features and problems of VSEs, (b) introduce our actual implementation "Simoland", and (c) report a study that investigates the influence of "real world" relationship satisfaction, intimacy motive, and autonomy motive on participants' behavior towards a virtual spouse who lives in Simoland.

\section{Features and Problems of Open Virtual Social Environments (VSEs)}

In the following section some general thoughts on the design of VSEs are made. An early decision has to be made concerning the technical realization. In the construction of a VSE, a trade-off exists between the technical effort ensuring a high fidelity of the virtual environment, for example with head-mounted displays or haptic feedback gloves, and the applicability of the computer test in terms of easiness, coverage, and interoperability on different systems. Recent research has shown that the amount of technical immersion is not necessarily connected to the subjective feeling of presence ("being there in the virtual world"). For example, visual realism does not seem to be a required factor for presence (Sanchez-Vives \& Slater, 2005), and ordinary PC monitors have been proven to work as well as a 150 -degree semi-immersive curved screen in a therapeutic setting (Tichon \& Banks, 2006). Concerning the interaction with virtual characters, humans are apparently capable of processing symbolic representations of people in almost the same manner as real ones, or as Sanchez-Vives and Slater (2005) state in their comment on a virtual exposure therapy of social anxiety: „Surprisingly, however, there is strong evidence that people respond to 
relatively crude virtual humans as if they were real people“" (p. 335).

Some problems arise in environments where the participants can freely choose where to go and what to. For example, in most environments the participant does not see the entire world at once, which poses the problem that the participant might miss information and events, which, however, are intended to be seen. Therefore, if special scenes are used to induce an experimental manipulation, it has to be made sure that the participant actually perceives the information. In the current implementation of Simoland, special "cut scenes" are used in such cases. Black bars appear on the top and bottom of the screen, and the player cannot interact with the other characters ("Simos") any more. Subsequently, all relevant Simos walk to pre-scripted positions where the scripted scene takes place as soon as all needed actors are on stage. In other cases, it becomes night (the screen gets dark in a rapid sundown), and on awakening all characters are at a new place where something important happens. Cut scenes of this kind can be used to reset the game to fixed states for all participants, introducing some amount of control and replicability in spite of the generally unrestricted nature of the setting.

However, cut scenes cannot control for the psychological carry-over effects that the participants may experience during the game. For example, if one participant starts a harsh dispute with the spouse while another participant is engaged in kissing and caressing, both will experience the next scene in a different light, even if a cut scene reseted the physical positions and environmental properties. This non-independence of behavioral acts is both an inherent, unavoidable feature and a possible problem of open environments; it reflects what happens in the process of real world behavior that is largely non-independent as well.

\section{What Can Be Measured in Virtual Social Environments?}

What is measured in virtual social environments is participants' behavior, just as in any other controlled setting. While behavior in most psychological studies serves as a dependent 
variable that is predicted by person factors (e.g. attitudes or motives) or situational factors, behavior can also serve as an independent variable that refers to internal states or traits of individuals. Accordingly, VSEs can be employed in two kinds of research questions: (a) What consequences do certain psychological characteristics and situational variations have on the behavior in VSEs, and (b) what tells us the behavior in VSEs about psychological constructs and real world behavior of the participants?

In the case of behavioral assessment as a psychological test, we would argue that the test operates at a semi-implicit level. While the labels "implicit-explicit" are quite common for the description of psychological measures, in fact several dimensions underlie this distinction (De Houwer, Teige-Mocigemba, Spruyt, \& Moors, 2009; Fazio \& Olson, 2003; Greenwald \& Banaji, 1995; McClelland, Koestner, \& Weinberger, 1989). Implicit measures can be defined as measurement outcomes that are produced "in the absence of certain goals, awareness, substantial cognitive resources, or substantial time" (De Houwer et al., 2009, p. 350). According to that definition and following the procedure of De Houwer et al. (2009), in the following section we analyse VSEs concerning their "implicitness" along several categories.

Fixed vs. free response set. While forced-choice Likert-type questionnaires on the one extreme have a completely fixed and restricted set of possible responses, a free association session in psychotherapy would mark the other extreme of a free response set. Only few assessment methods use a completely free response set, like free text production in TAT-like measures. In Simoland, the behavioral options are fixed. However, both the choice and the timing of these behaviors are unrestricted: Participants can choose how often they interact with their virtual spouse (they can even ignore her or him at all), and there are always more behavioral options available than can be enacted in a particular scene.

Presence of goals. Most psychological assessments measure cued responses 
where explicit tasks or social expectations are present concerning the "what" and "how" of behavior. On the other hand, implicit motive measures from the TAT-tradition, for example, focus on operant behavior (McClelland et al., 1989; Schultheiss, 2001), where the respondent is rather unrestricted concerning content, style and amount of responses. In our current study, participants were encouraged to play freely and to do just what they wanted. It was emphasized that no goal had to be achieved and that there was no "good" or "bad" behavior in the game.

Awareness of measurement. It makes a difference whether participants know that they are observed or not. Unaware participants are supposed to behave more authentically and unbiased. However, even if participants are aware of generally being observed, they sometimes do not know what exactly is in the focus of the research. Hence, they lack the specific awareness of the measurement procedure. While the general awareness of being observed certainly was present in Simoland, different game indices supposedly differ in becoming specifically aware. The participants probably know that certain choices of behavior are recorded, but we would argue that most participants are unaware if spacial distances to other Simos or viewing times are measured.

Controllability of behavior. Even if participants are aware that and how they are observed, and show a conscious or unconscious effort for impression management, that effort would only be consequential if the behavior in question can be under voluntary control. While self-reports and questionnaires are nearly arbitrarily manipulable, reaction times and even more physiological reactions are supposedly less prone to manipulation. But even if some behaviors could be perfectly controlled in under according instructions, humans usually do not do it all the time. There are several moderating factors that influence the actual amount of behavioral control (e.g. self awareness or cognitive load, Strack \& Deutsch, 2004). In Simoland, most - if not all - measured behavioral indices can be controlled if participants are 
instructed about how they work and how they should control their behavior.

Consequences of behavior. Many studies in social psychology investigate human behavior using vignettes of situations with a subsequent forced choice of several possible behaviors. One weakness of this widespread procedure of investigating self-reported hypothetical behavior is the lack of social consequences (Furr, 2009). In Simoland, at least some social consequences are present. For example, if the spouse is unduly criticized, he or she reacts angry, goes away, and is not in the mood for intimate interactions for some time. In a pretest of Simoland we asked participants about their experiences in the game and whether they experienced particular emotions. The majority of participants did so, and their answers in postexperimental interviews suggest a considerable impact of social consequences in the virtual world on them (e.g., "When my partner showed deeper feelings to the newcomer, it really shocked me. But I tried to convince him that I am the best for him!”).

To summarize, in virtual social environments like Simoland a variety of behavioral measures can be assessed. While the choice of behavioral options is more explicit, some other indices like spatial distances, time partitioning or viewing times are more implicit. While VSEs may not be as implicit as projective tests or pure reaction time measures, they certainly differ from self-declarative measures such as questionnaires.

\section{Implementation of "Simoland"}

In the implementation of Simoland we wanted to achieve an easy access for participants by keeping technical hurdles as low as possible. We therefore decided to implement a twodimensional game using the Adobe Flash technology (version 9). With that approach every participant can play the game using an Internet browser, the only requirement being an installed Flash Player plugin (which applies to over 98\% of internet users in Europe and the US, Adobe Systems Inc., 2009). The player's character, the virtual spouse, as well as the other characters in Simoland were displayed as rather simple organisms (see Figure 1). As 
argued above, visual realism is not a necessary prerequisite for a successful immersion, and keeping it visually simple also ensures an easy and flawless distribution of the game over the Internet. Moreover, theoretical reasons speak in favor of a rather symbolic approach. The theory of the 'uncanny valley' (Mori, 1982) suggests that believability rises with increasing realism. In some region short of $100 \%$ realism, however, users are jolted by some minor inconsistencies, which completely destroy the illusion of realism. We would argue that in the case of close relationships, this poses a particular problem because the detailed knowledge of the significant other (e.g., facial features) alerts participants to visual inconsistencies prevents successful immersion.

In a pretest of the current study, we indeed found such a pattern. Participants were equipped with an elaborated editor for modeling a rather realistic face of a person.

Participants were instructed to model an avatar of their real-life partner, which would be used in the subsequent computer game. They could adjust the hair-do, hair and skin color, the shape and position of mouth, nose, eyes, etc. The pretest, however, was quickly aborted because the participants expressed enduring concerns about the accurateness of the virtual avatar of their partner and often ruminated about details of the avatar that completely prevented immersion in the game. While in several scenarios increased realism indeed might have an impact on believability and immersion (e.g. Yee, Bailenson, \& Rickertsen, 2007), theoretical considerations and the experiences from our pretest led us to the conclusion that in the special case of a virtual spouse, "less is more". We think that a symbolic depiction of the virtual partner decreases feelings of enstrangeness and inconsistency, and rather provides a projection surface where behavioral and emotional characteristics of the real partner can be applied on.

Initially, the player's character was alone in Simoland, providing the possibility to explore interactions with inanimate objects (e.g., eating cakes, drinking water, or listening to 
an MP3 player) and to learn to control the game. After a few minutes, the player's virtual spouse and later some other inhabitants of Simoland ("Simos") were introduced. Our aim was to construct a VSE that is easily applicable to a wide range of testing situations, and as automated and controllable as possible. Because this approach rules out confederates controlling the other characters in the environment, they are implemented as autonomous agents.

Whenever the participant clicked on an object, a menu of possible actions appeared, depending on the kind of object. When clicking on another Simo, more than 30 different actions appeared (see Table 1). The autonomous agents reacted according to an underlying model that takes several internal states into account, such as familiarity, the mood of the character, or the type of the last interaction. Generally speaking, the agents followed a tit-fortat strategy. For illustration, we want to report a typical stream of interactions, which actually took place in the record of a male participant in the current study: The player approaches the spouse; he aks her to kiss him intensely; she refuses to do so; the player is pulling her legs; the spouse turns away, displaying mild anger; after $2 \mathrm{~min}$., the participant approaches the spouse again, making a compliment; the spouse reacts delighted; the participant asks for a small kiss; the spouse kisses him.

The game was kept as visual as possible (see Figure 1) with all reactions depicted by symbols, colors (e.g. a red glow and grey steam for angry Simos), or movements (e.g. turning away for disapproval). The only textual elements were some instructions and the labeling of the options one could choose.

-- INSERT FIGURE 1 ABOUT HERE

\section{Driving Forces of Behavior in Simoland}

A key principle of psychological assessment is "The best predictor of future behavior is past behavior". Accordingly, can we assume that "virtual behavior" is a valid predictor of 
"real behavior"? Some skeptics might state that behavior in computer games is completely arbitrary, or even that humans show the outright opposite reaction to what they would do in real life in order to safely experiment with other options. In contrast, however, many authors propose that participants' virtual behavior is correlated with real life motives, attitudes, and behavioral tendencies, as behavioral scripts, schemes, or internal working models about social interactions supposedly guide both real and fictitious interactions (Blascovich et al., 2002; Fincham \& Beach, 1988; Waters \& Waters, 2006). What are some of the forces that drive behavior in the current implementation of Simoland?

Interpersonal Motives. As motives are defined as concerns that drive, orient, and select behavior (McClelland, 1987), we expect motives to play a key role in the selection and energization of virtual behavior as well. Concerning motives in the interpersonal domain, many authors propose two broad categories, which are described as "Communion-Agency", "Love-Power", or “Affiliation-Dominance” (e.g. Horowitz, Wilson, Turan, Zolotsev, Constantino, \& Henderson, 2006). If these broad categories are applied to the case of romantic relationships, they can be termed as partner-related intimacy motive on the one hand, and partner-related autonomy motive on the other hand (Hagemeyer \& Neyer, 2009; Hmel \& Pincus, 2002; McAdams, Hoffman, Mansfield, \& Day, 1996). The partner-related intimacy motive describes the need for closeness to a romantic partner, which is indicated by, e.g., frequent self-disclosure.

Concerning the other dimension, unfortunately different psychological constructs share the same label "autonomy" (Hmel et al, 2002). In our study, autonomy is conceptualized as "reactive autonomy" (Koestner \& Losier, 1996). In this sense, highly autonomous individuals try to re-establish their independence if they experience a restriction of their freedom, or pressure from others. Hence, the need for autonomy in close relationships describes individuals who have a preference for experiencing individuality and independence from 
their partner, which, however, is not necessarily the opposite of experiencing intimacy. Therefore we conceptualize both motives as referring to orthogonal dimensions of interpersonal needs and behaviors. We expect that these motives play a key role in virtual relationship scenarios.

Presence. People differ on how strongly they get immersed into virtual realities and computer games, an experience called presence (Sanchez-Vives \& Slater, 2005). This "sense of being there" could moderate the relationship between real world properties and in-game behavior. On the one hand, some authors (e.g., Blascovich et al., 2002) argue that a higher amount of presence increases the realism of virtual behavior, which could lead to a more valid assessment of interindividual differences. On the other hand, a stronger feeling of "being there" could also foster efforts for impression management and deliberative processing, which in turn could lead to a more uniform, socially accepted behavior and a less valid assessment of interindividual differences.

Iterative Choices. One advantage of VSEs is the possibility of assessing behavioral choices in an ongoing relationship. The relational choices that we make in our relationships do not exist in isolation, but rather are embedded both in a history of past choices and experiences, and a future that is influenced by the choices made at the very moment. The focus on hypothetical "one-shot" situations, without any consequences for the real or imagined relationship, leads many studies to neglect this context. Furthermore, research on the prisoner's dilemma shows that choice behavior is entirely different between one shot situations and iterative games where one has to interact repeatedly with the same partner (Axelrod, 1984; Vicary \& Fraley, 2007). Vicary and Fraley (2007) already explored the evolution of relational choices in an imagined story with an ongoing relationship. While reading a short story about a relationship, participants encountered 20 choice points where they either had to choose a relationship enhancing alternative or a destructive alternative 
about how the story should continue. In three studies Vicary and Fraley (2007) analyzed the evolving choices within each participant, and found that the participants' attachment style had an influence on both the start of the imagined relationship and on the course of choices they took during the evolving story.

\section{The Current Study}

We chose to implement a similar design to Vicary and Fraley (2007) that, however, differed in three respects. First, our story is presented visually rather than verbally, as visual stimuli supposedly have a more direct access to implicit motives and intuitive reactions (Schultheiss, 2001). Second, no forced choices at defined points are required; instead, the participants can interact with their virtual spouse whenever they want and how often they want, providing a more naturalistic setting and facilitating spontaneous behavior. Third, behavioral choices are not restricted to a dichotomous choice; in fact more than 30 different actions are possible. Implementing a tit-for-tat strategy, the spouse always reacts accordingly to the player.

Based on the above considerations, we developed several specific hypotheses regarding the interplay of game behavior and real life variables. Concerning intimacy, numerous studies have shown that certain verbal and non-verbal behaviors (e.g., emotional self-disclosure, gentle touching, physical proximity) are triggered by the intimacy motive and in turn create a feeling of intimacy (for an overview, see Prager, 1995). The respective behaviors in Simoland are categorized under the label "positive behaviors" (see Table 1). As intimate interactions usually occur within already familiar relationships (Prager, 1995), we only expect these behaviors to occur towards the virtual spouse, which - at least concerning his or her formal status - has a higher familiarity than the other unknown Simos in the game. Hence, we propose the following hypothesis at the level of aggregated behavior:

H1a: Participants with a higher intimacy motive show more positive behavior and less 
negative behavior to their virtual spouse, whereas their behavior to other Simos is unrelated to this variable.

Likewise, relationship satisfaction is a predictor for positive behavior in a relationship (e.g., Gottman \& Levenson, 1992). As the virtual spouse is not introduced as an avatar of the real life partner, it may not be obvious that a good relationship satisfaction to the real life partner should predict behavior towards the so far unknown virtual spouse. Research on attachment, however, shows that experiences and mental representations from significant others are transferred from past relationships to new relationships, and also from past relationships to hypothetical relationships (Brumbaugh \& Fraley, 2006; Roisman, Collins, Sroufe, \& Egeland, 2005). Furthermore, research on the phenomenon of transference repeatedly has shown that relationships to significant others influence how interactions with new people unfold (Andersen \& Cole, 1990; Andersen \& Thorpe, 2009). This effect of transference, however, only takes place if the new person resembles the significant other in some minimal way. As physical features cannot account for this resemblance in the current setting (due to the symbolic depiction of the Simos), we expect that the mere relational status of the virtual spouse ("These two Simos have a romantic relationship", see also below) suffices to trigger mental representations of the real life partner. In extending these findings from attachment theory and transference to the virtual setting, we propose the following hypothesis at the level of aggregated behavior:

H1b: Participants with a higher relationship satisfaction show more positive behavior and less negative behavior to their virtual spouse, whereas their behavior to other Simos is unrelated to this variable.

The current implementation of Simoland is a very unrestricted setting, where 
participants can do what they want, without any pressure or external control from the instructions, the other Simos, or the virtual spouse. As in the current scenario no triggers for reactive autonomy (Koestner et al., 1996) are present, the autonomy motive is not supposed to be relevant for virtual behavior in Simoland. Hence, we hypothesized that:

H2: Participants' autonomy motive is unrelated to their behavior toward the virtual spouse or other Simos.

Concerning the course of interactions we expect a differentiated effect for the start and the course of the virtual relationship. According to the theories and research on transference, we expect the virtual character to be a sort of "projection screen", onto which expectations and behavioral patterns with the current real world partner are projected. Hence we propose that:

H3: Relationship satisfaction to the real world partner sets the initial level (i.e. the intercept) of interaction positivity.

During the course of interactions, however, motivational dynamics are supposed to shape behavior. Persistence as a key construct of motivational psychology describes the tendency to continue a behavior when no external pressures or requirements are present, even in the presence of obstacles or the absence of direct rewards (McClelland, 1987). This leads to our last hypothesis regarding the course of interactions:

H4: Participants high in intimacy motivation show a high persistence of close and positive behavior, and therefore show no decline of positive interactions over time, whereas participants low in intimacy motivation show a decline of positive interactions due to their lacking persistence. 
As no directional hypothesis could be derived from existing literature concerning the impact of presence, we additionally explored the moderating effect of presence on the effects expected by hypotheses H1-H4.

\section{Method}

\section{Participants}

Participants were recruited to participate in an online experiment advertised on the online portal of the Department of Psychology, Humboldt-University Berlin (www.psytests.de). The announcement of the study required participants to be at least 18 years old and to be currently involved in a serious, heterosexual relationship with a relationship duration of at least six months. After removal of participants who did not meet these requirements, 236 participants remained in the final data set. The average age was 32 years $(S D=11$; range 18-66 years), 189 participants were female. As an incentive for participation, participants received a personality profile based on their individual responses directly after the experiment.

\section{Procedure}

The study lasted for about 35 minutes and consisted of three parts. At the beginning, a priming procedure was presented to the participants. They either had to visualize a moment in their relationship in which they felt very close to their partner (intimacy prime), or they had to visualize a moment were they wanted to be alone (autonomy prime). However, as this priming procedure did not show statistical effects on any of the analyses below, it is ignored in the remainder of this article.

As second part of the study, the game took place. Participants were guided through a short tutorial (about 3-6 min.) on how to play the game. The tutorial introduced the setting of the game as "Simoland" which is inhabited by several "Simos". After these general 
informations, the player-controlled character (which was matched to the participant's sex) was introduced and it was shown how to control the player's character and how to start various interactions with inanimate objects and other Simos. Subsequently, the spouse of the player's character was introduced with the words: "These two Simos have a romantic relationship". Concerning the relationship between the participant's agent and its virtual spouse, we intentionally kept the instructions as short as possible. The idea of the game is to assess spontaneous and operant (McClelland et al., 1989) reactions. Hence, we tried to activate the conscious self-concept as little as possible. Throughout the game, it never was stated that the Simo represents the participant; instead, it was only pointed out that commands can be given to one of the Simos. Likewise, we did not state that the virtual spouse represents the real-life partner of the participant. Furthermore, in order to increase spontaneous relationship behavior, we did not give any instructions about how the participant should behave (e.g., we did not tell participants that they should treat the virtual spouse the way they would treat their real-life partner). Additionally, we instructed participants to play freely and that no goals are to be achieved. To increase the social significance of their actions, however, it was pointed out that every choice they made had an effect on the mood and the behavior of the other Simos and would affect their relationship. After the tutorial, the game itself started and lasted for about 14 minutes.

Third, the following questionnaires were assessed, among others:

\section{Closeness-Independence-Affiliation (CIA) Inventory (Asendorpf, Neberich, \&}

Hagemeyer, 2010). We only assessed the Closeness and the Independence subscales of this inventory. These short scales were designed to assess the motive for closeness to a romantic partner (intimacy motive), as well as the motive for independence and being alone (autonomy motive), on a 7-point Likert scale ranging from $1=$ never to $7=$ always. In previous studies these 8-item scales showed a good internal consistency (Cronbach's $\alpha>0.88$ ). 
Relationship satisfaction. As previous research indicates that single items can provide a pure and valid measure of relationship satisfaction (Doss, Rhoades, Stanley, \& Markman, 2009), we assessed relationship satisfaction to the real world partner with the following single item on a 11-point Likert scale ranging from $0=$ very unsatisfied to $10=$ very satisfied: "How satisfied are you all in all with your current relationship?".

Presence. After the game, participants were asked to rate their feeling of presence on four items with a 5 -point Likert scale ranging from $1=$ not at all to $5=$ very strong. Items have been adopted from existing presence questionnaires (Van Baren \& Ijsselsteijn, 2004) to fit to Simoland (e.g. "How strong was your sense of "being there" in the game", or "How aware were you of the real world surroundings while playing the game"; a full list of items can be obtained from the first author).

\section{Statistical Procedure}

Comparable to previous research in couple interactions (e.g. Gottman et al., 1992), all possible behavioral choices were a priori categorized into positive, neutral, and negative actions (see Table 1). We then calculated three game indices from aggregated behavioral choices towards the spouse: the ratio of positive choices to all choices (positivity), the ratio of negative choices to all choices (negativity), and the interaction frequency to the spouse divided by all interactions with other inhabitants of Simoland (spouse-directedness). Thus, all indices were standardized relative to the overall number of actions of each participant. Likewise, we calculated the positivity and the negativity index for interactions to all other Simos in the game.

Relationships between these game indices and personal characteristics of the participants were investigated with bivariate correlations. After transforming skewed variables by taking the inverse, logarithm, or square root, all but one variables still showed a significant deviation from normality ( $p$ values of the Shapiro-Wilk tests were $<.05$ ). As 
Pearson correlations of the transformed variables yielded virtually the same results as Spearman's rho, we decided to use this more robust measure of correlation.

For the analysis of the course of interactions with the virtual spouse we were interested in the changes in the probability of a positive (vs. negative) choice during the course of the game. Therefore all actions towards the spouse were a priori coded as positive $(=1)$ or negative $(=0)$. Each interaction had a time stamp serving as a predictor to assess a linear trend over time. As the number of interactions varied between participants, and the occurrences were not equally distributed in time, a multi-level modeling strategy was applied. Employing a hierarchical model, personal characteristics were used as level-2 predictors that explained random effects both in intercept and slope of the linear regression of each participant's interactions on time (level-1). As the interactions were represented as binary responses, they were analyzed using a generalized linear mixed model with a logit link function (Gelman \& Hill, 2007). The computational package lme4 (Bates, Maechler, \& Dai, 2009 ) in the R Environment for Statistical Computing (R Development Core Team, 2008) was employed to fit the model.

To summarize, dependent variable was the valence of each interaction (positive or negative), level-1 regression predictor was the time stamp of each interaction, normalized with regard to the duration of the game $(0=$ start of the game, $1=$ end of the game $)$. Participants were treated as random factors. Predictors on level-2 were the intimacy and the autonomy score as well as relationship satisfaction. Or, to put it in other words, we estimated a logistic regression for each participant to see whether there was a linear trend in time to show more or less positive interactions (level-1). Interindividual differences in the intercept (“How positive do they start?") and the slope ("Is there a decline or increase in positivity?") of this regression in turn are modeled by personality characteristics at level-2 (In generalized linear multilevel models, however, both steps are jointly estimated using a restricted 
maximum likelihood approach.).

In multilevel longitudinal models, it is usually assumed that the level-1 residuals are uncorrelated. In longitudinal models, however, one can often find an autoregressive structure among the residuals (Luhmann \& Eid, 2009; Rovine \& Walls, 2006) such that previous values of the dependent variable influence the current value. Hence, we controlled for a lag-1 autoregression by including an autoregressive parameter in our model so that the valence of a behavioral choice at time $t_{2}$ was predicted by the last choice at $t_{1}$. The autoregressive parameter was centered on the individual level.

\section{Results}

\section{Aggregated Behavior}

Participants initiated 28.32 interactions with their spouse on average $(S D=10.09)$. From all non-neutral interactions, only $7 \%$ were classified as negative, a finding consistent with Vicary and Fraley (2007) where many decision points showed negative choice rates below $10 \%$. Internal consistency of aggregated behavior was assessed by time-slicing the behavioral protocol into six equal slices and calculating the odd-even reliability for each game index (slicing into 4 or 12 slices yielded comparable results). The index positivity had an internal consistency of 0.18 , negativity 0.66 , and spouse-directedness 0.37 . However, as argued above, behavioral choices in an ongoing relationship are not independent observations. As the recorded behaviors violate the assumption of local item independence, the calculated odd-even reliability should not be necessarily seen as a property of the assessment method but rather as a statement about the stability of the underlying behavior in the context of the presented situations. Furthermore, investigations of the properties of Picture Story Exercises (like the TAT) show that a low internal consistency often is not an upper ceiling for validity (as assumed by classical test theory), but rather that low internal consistency can be accompanied by high validity (e.g., Schultheiss, Liening, \& Schad, 2008). 
To investigate the relation between in-game behavior and real world variables we correlated these game indices with the personality scales and relationship satisfaction. All test scores showed internal consistencies (Cronbach's $\alpha)>.73$, see Table 2 . As the game indices showed a highly skewed distribution we used Spearman's rho as an index of correlation. All behavioral indices towards the spouse significantly correlated with two of the assessed personality variables (see Table 2): Participants high in intimacy motivation and with a high relationship satisfaction showed more interactions with the virtual spouse overall, more positive interactions, and fewer negative interactions. The autonomy motive yielded no significant correlations, as predicted.

Concerning the behavior to other Simos, only two coefficients were significant: Participants with a higher spouse-directedness showed a lower ratio of positive (but not more negative) behaviors towards other Simos. Furthermore, participants with a higher relationship satisfaction with their real life partner showed a lower ratio of positive interactions to others. Hence, the relationship-related variables like intimacy motive and relationship satisfaction only correlated with spouse-directed behavior in the expected direction.

To test whether the regression with spouse-directed behavior continued to be significant if other-directed behavior was taken into account, we ran additional multiple regression analyses where the spouse-directed behavior was controlled for the other-directed behavior. In these analyses, the results were virtually identical with the results without controls.

-- INSERT TABLE 2 ABOUT HERE --

\section{Course of Interactions}

For the investigation of the time course of behavioral choices, we analyzed intraindividual changes of positive vs. negative choices over time. Unit of analyses were 3042 observable positive or negative behaviors nested in the interactional records of 236 participants. Therefore each participant's change in behavior was assessed with 13 data points 
on average, allowing an assessment of intraindividual change based on many assessments. Since the dependent variable was the binary outcome of a positive (vs. negative) interaction with the virtual spouse, the intercept in the model refers to the probability of a positive interaction at the beginning of the game, while the slope refers to a linear trend of a declining or increasing probability of a positive interaction during the course of the game (logistic curve).

The best model fit was achieved by allowing both intercepts and slopes of the level-1 predictor to vary across participants. In the model, intercept variance was 6.71 and slope variance was 11.42. Compared to the unconditional model, level-2 predictors explained 11\% of the variance both in intercepts and slopes. In the following we concentrate on the fixed effects of the model (see Table 3).

\section{-- INSERT TABLE 3 ABOUT HERE --}

The significant effect of relationship satisfaction on the intercept shows that participants who were satisfied with their real world relationship started the game with more positive behaviors. In contrast, the time trend of behavior was only affected by the intimacy score; participants with a higher intimacy motive tended to keep or slightly increase their positivity, while a low intimacy motive led to a decrease in positivity (see Figure 2).

Concerning autoregression, a significantly positive coefficient was estimated. Hence, there is some behavioral stability in the stream of behavior, as the positivity of the current behavior matches the last behavior with a probability greater than zero ${ }^{1}$.

\section{-- INSERT FIGURE 2 ABOUT HERE --}

\section{Moderating Effect of Presence}

For testing the possible moderating effect of presence on bivariate correlations we

\footnotetext{
${ }^{1}$. We also tested a lag-2 and a lag-3 autoregression. These parameters, however, neither were significant, nor did their inclusion change the coefficients of the other predictors.
} 
conducted hierarchical multiple regressions with products of the standardized predictors as interaction terms (Aiken \& West, 1991). The interaction terms with the presence score did never reach significance, indicating that felt presence does not moderate the relationship between personality variables and aggregated game indices ${ }^{2}$.

Concerning the course of interactions, we added a main effect for presence, as well as interaction terms between presence and all other level-2 predictors into the model. Neither the main effect for presence nor the interaction terms including presence reached significance (see Table 3). Therefore the amount of felt presence did not moderate the course of interactions in general, nor the between-participant effects.

\section{Discussion}

The aim of the current article was to demonstrate the potential of virtual social environments for psychological assessment with the actual implementation "Simoland". We investigated the relationship between the behavior towards a virtual spouse and real-life variables (i.e., interpersonal motives and relationship satisfaction). We tested four hypotheses on aggregated behavior, the initial state and the dynamics of virtual spouse interactions, and found support for all of them, providing one of the first evidence that behavior in virtual social environments is not completely arbitrary but is correlated with "real life". To clarify, we do not assume that participants think that the virtual spouse represents the real partner, but rather that stable emotional and behavioral schemes (i.e., the interpersonal motives), as well as expectations from the current relationship are transferred to the virtual spouse.

In our study, participants treated the virtual spouse according to their personality and their experiences in the current real life relationship. Our results suggest that participants

\footnotetext{
${ }^{2}$. Concerning non-normality, we ran moderation analyses both with untransformed variables and with the best possible transformation applied to the skewed variables. The interaction terms stayed insignificant, regardless of transformation.
} 
transfer their experiences and expectations from their real relationship into the virtual world. Participants who are less satisfied with their real relationship start less positive into the virtual relationship, perhaps re-instantiating their current mode of relationship in the virtual environment. During the game, however, the behavior of the participants gets more and more shaped by their intimacy motive: Only participants high in intimacy motivation continue to show and even increase the ratio of positive and close interactions. This reflects the effect of behavioral persistence in highly motivated persons (McClelland, 1987). Furthermore, these results could only be found concerning spouse-directed behavior - behavior to other Simos does not show these patterns. This finding adds to the validity of Simoland, as indeed partner specific behavioral tendencies are expressed (and not a general tendency towards all virtual agents). The only significant (but low) correlation between real world variables and otherdirected behavior was an unexpected increase of positive behavior towards others in participants with a low real life relationship satisfaction. A possible interpretation of this result could be that participants with a dissatisfying real life relationship project their negative expectations on the virtual spouse, and rather try to establish close and positive interactions with other Simos.

The analysis of the process of interactions highlights another strength of the approach: While the intimacy motive and relationship satisfaction showed comparable correlational patterns concerning aggregated behavior, both could be dissociated in the process analysis of behavior. Although both constructs showed a substantial correlation at the aggregate level ( $r$ $=.42$ ), the analysis of interactions revealed that both are not the same. While relationship satisfaction sets an initial bias for interactions, motivational dynamics unfold over time, supporting the usefulness of time-course analyses for the investigation of motivational and interactional processes. Due to the unrestricted nature of the setting, we hypothesized that participants with a high need of autonomy had no need of breaking free from any restrictions 
(i.e., there was no instigating situation for the autonomy motive to get active). As predicted, the autonomy motive did not have an influence on behavior in this study. One of the reviewers, however, pointed to an alternative explanation for this null result: it could be that participants with a high autonomy motivation expressed their autonomy by breaking off from the study, which would have caused a restriction of variance. We assessed the autonomy motive after the game, hence we cannot test for a selective drop out. While we cannot rule out this alternative explanation, we would argue that participants had no need to break off from the study, as the nature of the setting was more unrestricted than in most other psychological studies. Furthermore, participants could have easily expressed their autonomy within the game, by not interacting with the spouse, keeping the distance, or engaging in flirting or conversation with others.

The analysis of the moderating role of presence showed no differences between participants with high and low presence. Therefore, if VSEs are to be employed as diagnostic tools in the future, we provided preliminary evidence that their validity does not seem to be affected by the attitude of how participants approach and experience the game. Furthermore, these findings are the first to demonstrate the effect of transference towards a virtual relationship. This effect might be employed to develop new assessment methods that operate on a projective level, where unconscious internal working models and mental representations are applied on a virtual agent.

An increasing number of papers in psychology emphasize the importance of observing actual behavior in contrast to hypothetical choices or self-reported intentions (e.g., Baumeister, Vohs, \& Funder, 2007; Furr, 2009). Furthermore, other researchers have a strong distrust in self-report measures and argue that the observation of interactional processes is the key for understanding relationship outcomes (Gottman, 1998). Virtual environments can be useful tools for the generation and observation of actual behavior. One major obstacle, 
however, is the immense effort of setting up the virtual environments. Although we chose to implement a rather simple two-dimensional game, it took a long time to get it run smoothly. However, data analysis was relatively simple, as we did not have to go through hours of video coding, which means a shift in efforts from data coding to the preparation of the study. As another advantage, coding of behavior is unambiguous and straightforward, as all relevant behaviors have been classified a priori and one has not to deal with problems like interobserver reliability drift or decay (Gottman, 1998). Now that Simoland has been implemented it is relatively simple to construct new scenarios and experimental variations ${ }^{3}$. Furthermore, the computer game has not necessarily to be built up from scratch like we did. Depending on the research question, existing computer games with scripting ability could be employed (Frey, Hartig, Ketzel, Zinkernagel, \& Moosbrugger, 2007).

\section{Limitations of the study}

Both the theoretical considerations as well as the study itself have some limitations. Concerning empirical data of the current study, a considerable limitation is the uneven ratio of gender. With $80 \%$ female participants it is questionable whether results also generalize to a broader underlying population. Due to the low power resulting from the small sample size of men, separate correlational analyses of both genders are not very expedient. Adding gender as another level-2 predictor to the multilevel model of interactions, however, neither resulted in a significant coefficient nor did it alter the other coefficients, which provides at least preliminary evidence that the results are valid for both genders.

In the introduction, we argued that VSEs have the potential to measure psychological properties on a rather implicit level. A potential limitation of the the current study design is the reliance on self-report measures as validating criteria. Hence, it is hard to assess whether

\footnotetext{
${ }^{3}$. Researchers interested in using Simoland for their own research are encouraged to contact the first author.
} 
the game indices indeed have implicit properties. While the introduction tried to focus on general ideas and properties of open virtual social environments (VSEs), the degrees of freedom in the construction of such environments are so numerous that it might be inappropriate to subsume different implementations under a common label. One has to take a close look on each single implementation of a VSE (e.g. which scenarios are presented? Are specific goals present for the participant or is it a rather free exploration of the world? Are certain motives activated or not? How is the behavior of other characters in the game modeled?). Furthermore, although we argue that virtual behavior is actual behavior, these records of virtual behavior lack many indices of nonverbal and uncontrollable behavior investigated in other studies (like tenseness of the body posture; e.g. Asendorpf, Banse, \& Mücke, 2002). Therefore virtual environments will never replace observational studies in the lab or in the field, but rather complement them. The advantages of virtual environments become apparent in testing situations that rule out or complicate a lab setting, like embarrassing interpersonal situations with sexual content, unethical experimental manipulations, like arranging an extramarital affair for the partner, or the testing of participants who are living far apart, cannot move (e.g., prisoners), or have rare characteristics such that they are scattered across the country. The playful character of the game also could make it suitable for studying children's reactions in social situations.

\section{Future Studies}

In the current study, we did not construct a specific scenario but rather provided an open environment to be freely explored by the participants. We investigated the basic properties of VSEs - future studies should progress by addressing theory-driven hypotheses and by modeling specific situations like the induction of conflicts, or attachment related separation scenes. Additionally, one could model specific reaction styles from the virtual spouse. Furthermore, future studies should incorporate other measures that broaden the scope 
of correlates of virtual behavior: real-life-outcomes, behavioral observations in laboratory setting, diary data, or implicit measures like an IAT. In any way, we believe that the use of virtual social environments and computer games is a promising and viable way for the study of social interactions beyond self-report. 


\section{References}

Adobe Systems Inc. (2009). Adobe Flash Player version penetration. Retrieved Aug 24, 2009 ,

http://www.adobe.com/products/player_census/flashplayer/version_penetration.html.

Aiken, L. S., \& West, S. G. (1991). Multiple regression: Testing and interpreting interactions. Thousand Oaks, CA: Sage Publications.

Andersen, S., \& Cole, S. (1990). "Do I know you?": The role of significant others in general social perception. Journal of Personality and Social Psychology, 59, 384-399.

Andersen, S. M., \& Thorpe, J. S. (2009). An IF-THEN theory of personality: Significant others and the relational self. Journal of Research in Personality, 43, 163-170.

Asendorpf, J. B., Banse, R., \& Mücke, D. (2002). Double dissociation between implicit and explicit personality self-concept: The case of shy behavior. Journal of Personality and Social Psychology, 83, 380-393.

Asendorpf, J. B., Neberich, W., \& Hagemeyer, B. (2010). Closeness-Independence-Affiliation (CIA) Inventory. Unpublished scales. Humboldt University, Berlin, Germany.

Axelrod, R. (1984). The evolution of cooperation. New York: Basic Books.

Bates, D., Maechler, M., \& Dai, B. (2009). lme4: Linear mixed-effects models using S4 classes (R package version 0.999375-32 ) [Computer software].

Baumeister, R. F., Vohs, K. D., \& Funder, D. C. (2007). Psychology as the science of selfreports and finger movements: Whatever happened to actual behavior. Perspectives on Psychological Science, 2, 396-403.

Blascovich, J., Loomis, J., Beall, A. C., Swinth, K. R., Hoyt, C. L., \& Bailenson, J. N. (2002). Immersive virtual environment technology as a methodological tool for social psychology. 
Psychological Inquiry, 13, 103-124.

Brumbaugh, C., \& Fraley, R. (2006). Transference and attachment: How do attachment patterns get carried forward from one relationship to the next? Personality and Social Psychology Bulletin, 32, 552-560.

De Houwer, J., Teige-Mocigemba, S., Spruyt, A., \& Moors, A. (2009). Implicit measures: A normative analysis and review. Psychological Bulletin, 135, 347-368.

Doss, B. D., Rhoades, G. K., Stanley, S. M., \& Markman, H. J. (2009). The effect of the transition to parenthood on relationship quality: An 8-year prospective study. Journal of Personality and Social Psychology, 96, 601-619.

Fazio, R. H., \& Olson, M. A. (2003). Implicit measures in social cognition research: Their meaning and uses. Annual Review of Psychology, 54, 297-327.

Fincham, F. D., \& Beach, S. R. H. (1988). Attribution processes in distressed and nondistressed couples: 5. Real versus hypothetical events. Cognitive Therapy and Research, 12, 505-514.

Frey, A., Blunk, H. A., \& Banse, R. (2006). Psi-Land: Paarinteraktionsforschung in einer virtuellen Umgebung [Psi-Land: Investigating interactive behaviour in romantic couples in a virtual environment.]. Zeitschrift für Sozialpsychologie, 37, 151-159.

Frey, A., Hartig, J., Ketzel, A., Zinkernagel, A., \& Moosbrugger, H. (2007). The use of virtual environments based on a modification of the computer game Quake III Arena (R) in psychological experimenting. Computers in Human Behavior, 23, 2026-2039.

Furr, R. M. (2009). Personality psychology as a truly behavioural science. European Journal of Personality, 23, 369-401.

Gelman, A., \& Hill, J. (2007). Data analysis using regression and multilevel/hierarchical 
models. New York: Cambridge University Press.

Gottman, J. M. (1998). Psychology and the study of marital processes. Annual Review of Psychology, 49, 169-197.

Gottman, J. M., \& Levenson, R. W. (1992). Marital processes predictive of later dissolution: Behavior, physiology, and health. Journal of Personality and Social Psychology, 63, 221233.

Greenwald, A. G., \& Banaji, M. R. (1995). Implicit social cognition: Attitudes, self-esteem, and stereotypes. Psychological Review, 102, 4-27.

Greenwald, A. G., McGhee, D. E., \& Schwartz, J. L. K. (1998). Measuring individual differences in implicit cognition: The Implicit Association Test. Journal of Personality and Social Psychology, 74, 1464-1480.

Hagemeyer, B., \& Neyer, F. (2009). The Operant Motive Test for partner relationships (OMT_P): A new implicit measure of the partner-related needs for intimacy and autonomy. Manuscript submitted for publication.

Hmel, B. A., \& Pincus, A. L. (2002). The meaning of autonomy: On and beyond the interpersonal circumplex. Journal of Personality, 70, 277-310.

Horowitz, L. M., Wilson, K. R., Turan, B., Zolotsev, P., Constantino, M. J., \& Henderson, L. (2006). How interpersonal motives clarify the meaning of interpersonal behavior: A revised circumplex model. Personality and Social Psychology Review, 10, 67-86.

Koestner, R., \& Losier, G. F. (1996). Distinguishing reactive versus reflective autonomy. Journal of Personality, 64, 465-494.

Luhmann, M., \& Eid, M. (2009). Does it really feel the same? Changes in life satisfaction following repeated life events. Journal of Personality and Social Psychology, 97, 363-381. 
McAdams, D. P., Hoffman, B. J., Mansfield, E. D., \& Day, R. (1996). Themes of agency and communion in significant autobiographical scenes. Journal of Personality, 64, 339-377.

McCall, C., Blascovich, J., Young, A., \& Persky, S. (2009). Proxemic behaviors as predictors of aggression towards Black (but not White) males in an immersive virtual environment. Social Influence, 4, 138-154.

McClelland, D. C., Koestner, R., \& Weinberger, J. (1989). How do self-attributed and implicit motives differ? Psychological Review, 96, 690-702.

McClelland, D. C. (1987). Human motivation. New York: Cambridge University Press.

Mori, M. (1982). The Buddha in the robot. Charles E. Tuttle Co.

Prager, K. J. (1995). The psychology of intimacy. New York: Guilford Press.

R Development Core Team. (2008). R: A Language and Environment for Statistical Computing. Vienna, Austria: Basic Books.

Roisman, G., Collins, A., Sroufe, A., \& Egeland, B. (2005). Predictors of young adults' representations of and behavior in their current romantic relationship: Prospective tests of the prototype hypothesis. Attachment \& Human Development, 7, 105-121.

Rovine, M. J., \& Walls, T. A. (2006). A multilevel autoregressive model to describe interindividual differences in the stability of a process. In T. A. Walls \& J. L. Schafer (Eds.), Models for intensive longitudinal data (pp. 124-147). New York: Oxford University Press.

Sanchez-Vives, M. V., \& Slater, M. (2005). From presence to consciousness through virtual reality. Nature Reviews Neuroscience, 6, 332-339.

Schultheiss, O. C. (2001). An information processing account of implicit motive arousal. In M. L. Maehr \& P. Pintrich (Eds.), Advances in motivation and achievement (Vol. 12: New 
directions in measures and methods) (pp. 1-41). Greenwich, CT: JAI Press.

Schultheiss, O. C., Liening, S. H., \& Schad, D. (2008). The reliability of a Picture Story Exercise measure of implicit motives: Estimates of internal consistency, retest reliability, and ipsative stability. Journal of Research in Personality, 42, 1560-1571.

Strack, F., \& Deutsch, R. (2004). Reflective and impulsive determinants of social behavior. Personality and Social Psychology Review, 8, 220-247.

Tichon, J., \& Banks, J. (2006). Virtual reality exposure therapy: 150-Degree screen to desktop PC. CyberPsychology \& Behavior, 9, 480-488.

Van Baren, J., \& Ijsselsteijn, W. (2004). Measuring presence: A guide to current measurement approaches. OmniPres project IST-2001-39737. Retrieved Feb 28 2007, from http://www.presence-research.org/omnipres/D5.pdf

Vicary, A. M., \& Fraley, R. C. (2007). Choose your own adventure: Attachment dynamics in a simulated relationship. Personality and Social Psychology Bulletin, 33, 1279-1291.

Waters, H. S., \& Waters, E. (2006). The attachment working models concept: Among other things, we build script-like representations of secure base experiences. Attachment and Human Development, 8, 185-197.

Yee, N., Bailenson, J., \& Rickertsen, K. (2007). A meta-analysis of the impact of the inclusion and realism of human-like faces on user experiences in interfaces. Proceedings of the SIGCHI conference on Human factors in computing systems, 1-10. 
Table 1: Possible Interactions in Simoland

Positive behaviors

talk about one's mood, talk about a joint future, talk about the relationship, kiss (in three variants: short kiss, romantic kiss, familiar kiss), "tell me how you feel", hear music jointly, dance together, make a compliment, say "I love you!", caress, smooch, tell a vision: having a family, lifelong love, meeting a soulmate

Neutral behaviors

talk about hobbies, talk about occupational successes, gossip about other people, to turn sb. on (by showing off), call sb. to come, tell a vision: climbing a big mountain, being rich and successful, doing a world trip (alone)

Negative behaviors

"go away - I want to be for myself", send away (angry), start an argument, criticize, insult, ridicule, annoy 
Table 2: Spearman Correlations between Game Indices and Personality Variables

\begin{tabular}{|c|c|c|c|c|c|c|c|c|}
\hline Measure & 1 & 2 & 3 & 4 & 5 & 6 & 7 & 8 \\
\hline $\begin{array}{l}\text { 1. Positivity } \\
\text { (spouse) }\end{array}$ & - & & & & & & & \\
\hline $\begin{array}{l}\text { 2. Negativity } \\
\text { (spouse) }\end{array}$ & $-.30 * * *$ & - & & & & & & \\
\hline $\begin{array}{l}\text { 3. Spouse- } \\
\text { directedness }\end{array}$ & .06 & $-.19 * *$ & - & & & & & \\
\hline $\begin{array}{l}\text { 4. Positivity } \\
\text { (others) }\end{array}$ & -.02 & .02 & $-.23 * * *$ & - & & & & \\
\hline $\begin{array}{l}\text { 5. Negativity } \\
\text { (others) }\end{array}$ & .04 & .10 & .09 & $-.21 * *$ & - & & & \\
\hline 6. Presence & -.11 & .08 & .11 & -.05 & .03 & .73 & & \\
\hline $\begin{array}{l}\text { 7. Intimacy } \\
\text { motive }\end{array}$ & $.15^{*}$ & $-.29 * * *$ & $.22 * * *$ & -.05 & -.02 & .04 & .81 & \\
\hline $\begin{array}{l}\text { 8. Autonomy } \\
\text { motive }\end{array}$ & -.08 & .05 & -.04 & -.08 & .02 & .11 & $-.35^{* * *}$ & .82 \\
\hline $\begin{array}{l}\text { 9. relationship } \\
\text { satisfaction (real } \\
\text { life partner) }\end{array}$ & $.15^{*}$ & $-.20 * *$ & $.16^{*}$ & $-.14^{*}$ & .05 & .00 & $.42 * * *$ & $-.13 *$ \\
\hline
\end{tabular}

Note. Values in the diagonal are Cronbach's $\alpha$ (where applicable)

$* p<.05 . * * p<.01 . * * * p<.001$. 
Table 3: Generalized Linear Multilevel Model of Interaction Positivity: Fixed Effects

\begin{tabular}{lccc}
\hline Predictor & Estimate $($ logits $)$ & SD & $\mathrm{p}$ \\
\hline Intercept & 4.10 & 0.33 & $<.001$ \\
Intercept x autoregression & 0.54 & 0.20 & .008 \\
Intercept x relationship satisfaction & 0.72 & 0.34 & .034 \\
Intercept x intimacy motive & -0.33 & 0.37 & .369 \\
Intercept x autonomy motive & -0.18 & 0.35 & .609 \\
Intercept x presence & 0.34 & 0.33 & .294 \\
$\quad-0.97$ & 0.46 & .034 \\
Time & -0.82 & 0.49 & .093 \\
Time x relationship satisfaction & 1.19 & 0.52 & .023 \\
Time x intimacy motive & 0.22 & 0.49 & .658 \\
Time x autonomy motive & -0.34 & 0.46 & .460 \\
Time x presence & & & \\
\hline
\end{tabular}


Figure captions

Figure 1. Four exemplary scenes from Simoland. The angular shaped character with the triangle on its back is the agent controlled by the participant. All other characters are autonomous agents. From top left to bottom right: (a) talking about hobbies, (b) a gathering of Simos engaged in different activities, (c) "I love you", (d) a dispute.

Figure 2. Logistic slopes of the final GLMM. Logits are transformed to probabilities to ease interpretation. Logistic curves are plotted at $+/-1 S D$ of intimacy motive and relationship satisfaction. Other predictors are set to zero (i.e. to the sample mean), except the autoregression parameter, which was set to the individual mean. 


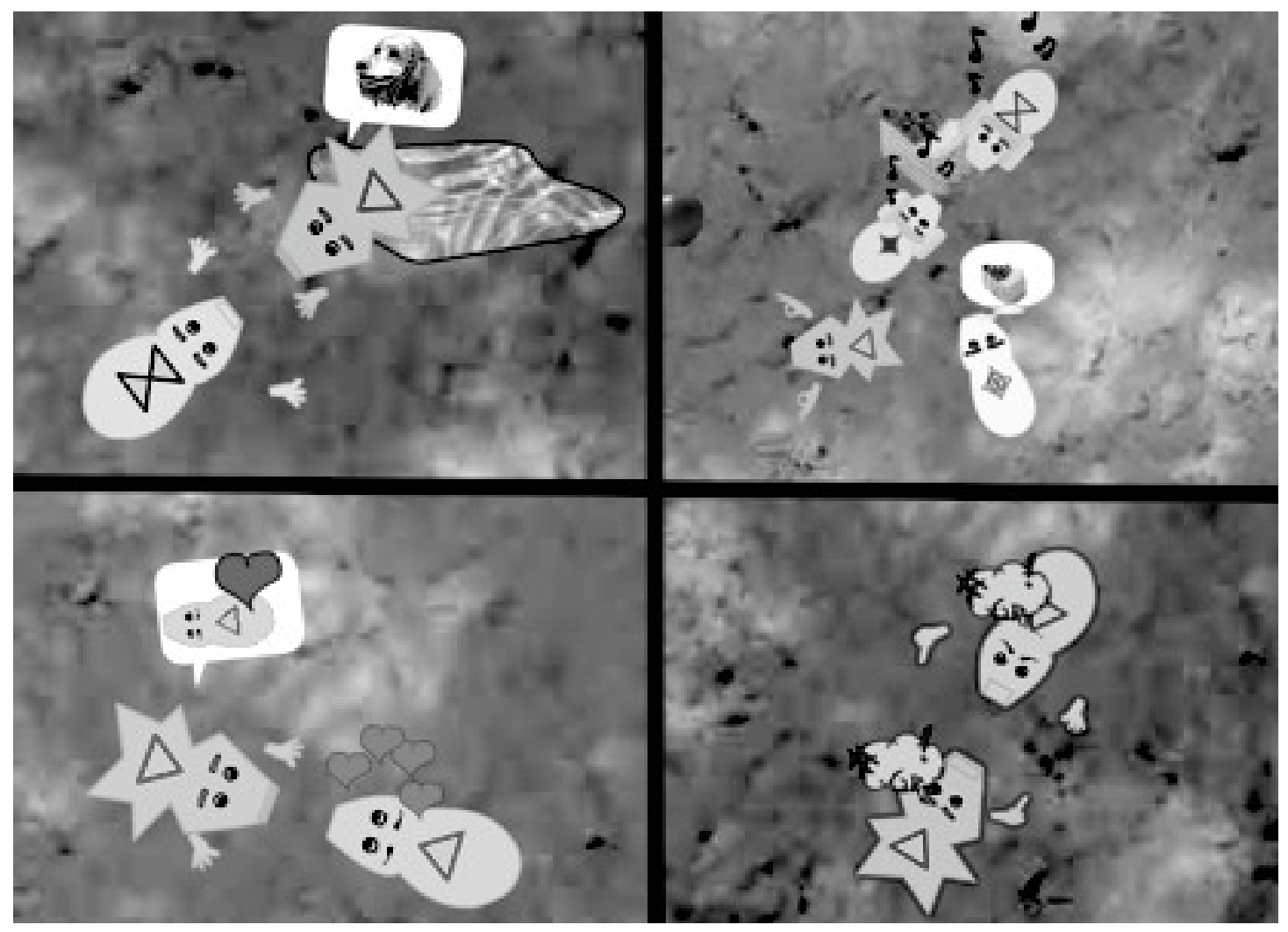




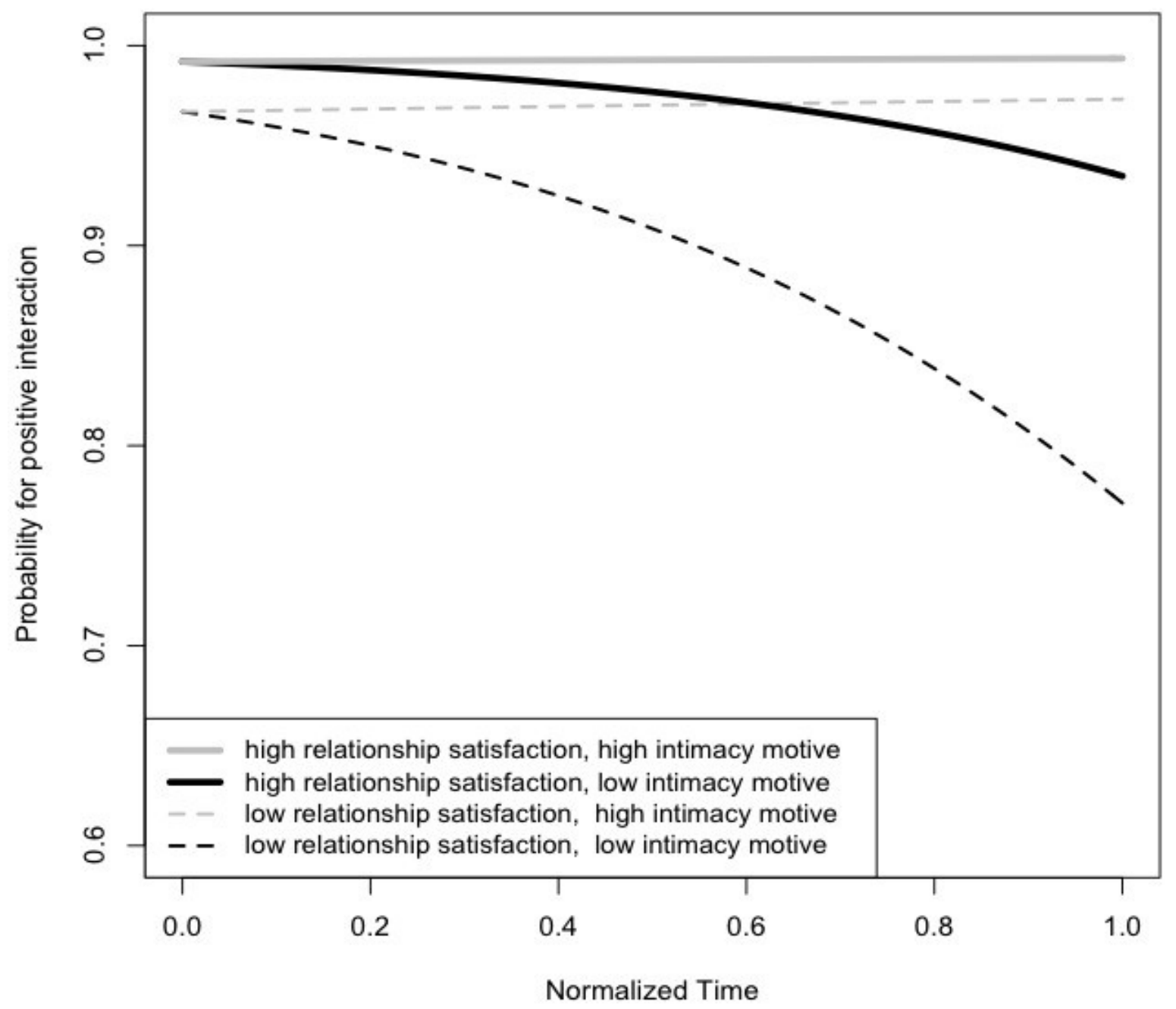

